\title{
Risk Factors of Delayed Recovery of Gastrointestinal Function After lleostomy Reversal for Rectal Cancer Patients
}

\author{
Lili Chu (D) ${ }^{1, *}$ \\ Hui Wang ${ }^{1-3, *}$ \\ Suyu Qiu ${ }^{4, *}$ \\ Biyan Shao' \\ Jia Huang' \\ Qiyuan Qin ${ }^{1-3}$ \\ Yanjiong $\mathrm{He}^{\prime}$ \\ Jing Xue ${ }^{5}$ \\ Xiaoyan $\mathrm{Li}^{5}$ \\ Xiaoyan Huang ${ }^{1-3}$ \\ Rongkang Huang (D) ${ }^{1-3}$ \\ 'Department of Colorectal Surgery, The \\ Sixth Affiliated Hospital, Sun Yat-sen \\ University, Guangzhou, 51 0655, Guangdong, \\ People's Republic of China; ${ }^{2}$ Guangdong \\ Institute of Gastroenterology, The Sixth \\ Affiliated Hospital, Sun Yat-sen University, \\ Guangzhou, 510655, Guangdong, People's \\ Republic of China; ${ }^{3}$ Guangdong Provincial \\ Key Laboratory of Colorectal and Pelvic \\ Floor Diseases, The Sixth Affiliated Hospital, \\ Sun Yat-sen University, Guangzhou, 510655, \\ Guangdong, People's Republic of China; \\ ${ }^{4}$ Department of General Surgery, Shunde \\ New Rongqi Hospital, Foshan, Guangdong, \\ 510655, People's Republic of China; \\ ${ }^{5}$ Department of Pharmacy, The Sixth \\ Affiliated Hospital, Sun Yat-sen University, \\ Guangzhou, 510655, Guangdong, People’s \\ Republic of China
}

*These authors contributed equally to this work

Correspondence: Xiaoyan Huang; Rongkang Huang

Department of Colorectal Surgery, Sun

Yat-sen University Sixth Affiliated

Hospital, No. 26 Yuancun Erheng Road,

Guangzhou, 510655, Guangdong, People's

Republic of China

Fax +86 020-38737621

Email huangxy278@mail.sysu.edu.cn;

huangrk3@mail.sysu.edu.cn
Purpose: The aim of this study was to identify the risk factors associated with delayed recovery of gastrointestinal function after ileostomy reversal for rectal cancer patients.

Methods: In this retrospective study, the data of rectal cancer patients who underwent ileostomy reversal from January 2018 to December 2019 at the Sixth Affiliated Hospital of Sun Yat-sen University were assessed to investigate potential risk factors of delayed flatus after ileostomy reversal.

Results: A total of 282 patients were eligible for this study. Postoperative first flatus time ranged from 1 to 9 days, of which $58.8 \%$ patients presented with delayed flatus that was longer than 3 days. Univariate analysis showed that delayed postoperative flatus was significantly associated with the length of postoperative hospital stay $(P<0.001)$ and postoperative complications $(P=0.037)$. Multivariate analysis showed that intravenous fluid infusion at postoperative day 1 (POD1) $(\mathrm{OR}=1.001,95 \% \mathrm{CI}: 1.001-1.002, P=0.001)$ and duration of stoma $\geq 6$ months $(\mathrm{OR}=2.005,95 \% \mathrm{CI}: 1.155-3.657, P=0.014)$ were independent risk factors for delayed flatus.

Conclusion: Increased intravenous fluid infusion at POD1 and duration of stoma $\geq 6$ months were related to delayed recovery of gastrointestinal function after ileostomy reversal for rectal cancer patients.

Keywords: ileostomy reversal, gastrointestinal function, rectal cancer

\section{Introduction}

Colorectal cancer is the third-most prevalent cancer and the second-leading cause of death worldwide, with 1.93 million new cases and 930,000 deaths in 2020, ${ }^{1}$ of which rectal cancer accounted for $44 \%$ of these cases. ${ }^{2,3}$ Surgical resection is the main treatment for rectal cancer and being a high-risk procedure, it is often accompanied by serious complications. ${ }^{4,5}$ Anastomotic leakage (AL) is a common but serious complication of rectal cancer and is associated with increased hospital stay, additional medical expenses, reduced survival, and unexpected early death of rectal cancer patients. ${ }^{4}$ To reduce the risk of $\mathrm{AL}$, or at least to reduce complications of anastomotic dehiscence, a temporary defunctioning stoma is often required. ${ }^{6-8}$

However, temporary defunctioning stoma like temporary ileostomy requires a second surgery to restore intestinal continuity and may increase patients' discomfort, length of hospital stay, and treatment costs. It is also associated with postoperative morbidities, such as postoperative intestinal obstruction, incision infection, AL, intestinal perforation and peritonitis, in up to $20 \%$ of the 
patients. ${ }^{9,10}$ Among these complications, delayed gastrointestinal function recovery (GIFR) could lead to accumulation of gas and effusion in the gastrointestinal tract lumen and increases the likelihood of intestinal obstruction and dysregulation of the gastrointestinal flora. The main clinical manifestations of delayed GIFR are delayed flatus or defecation, abdominal pain, abdominal distension, nausea, vomiting, and postoperative intestinal obstruction which often require nasogastric tube intubation. ${ }^{10-12}$ Delayed GIFR also affects the time for early oral nutrition, leading to insufficient nutritional supply and time for adjuvant treatment, thus, influencing treatment outcomes and patients' survival. ${ }^{13}$

GIFR and tolerance to oral diet are among the important criteria for postoperative discharge for rectal cancer patients. ${ }^{14}$ GIFR has been shown to affect the time for ileostomy reversal as inadequate recovery can lead to an increase in hospital stay of up to 23 days. ${ }^{12}$ Further, it was reported that intravenous fluid infusion, early enteral nutrition, procedure type, choice of postoperative analgesia, and surgery-induced stress response, was related to GIFR after ileostomy reversal surgery. ${ }^{15-17}$ However, few studies have investigated the risk factors for delayed GIFR after ileostomy reversal.

In this study, we aimed to identify the risk factors associated with GIFR after ileostomy reversal of rectal cancer patients for early clinical intervention in advance after ileostomy reversal in high-risk patients.

\section{Methods}

\section{Patients and Data Collection}

The data of rectal cancer patients who underwent ileostomy reversal surgery from January 2018 to December 2019 at the Sixth Affiliated Hospital, Sun Yat-sen University (Guangzhou, P. R. China) were retrospectively retrieved.

The data retrieved included demographics and clinical data, such as age, gender, drinking history, smoking status, concomitant disease, tumor recurrence or metastasis, BMI, pTNM stages of the primary tumor, distance from the tumor to the anus, chemotherapy, radiotherapy, surgical approach of primary tumor resection, duration of stoma $\geq 6$ months, operation duration of ileostomy reversal, methods of anastomosis, intravenous fluid infusion during ileostomy reversal surgery, intravenous fluid infusion in POD1, first postoperative flatus time, length of postoperative hospital stay, complications after primary procedure. The study exclusion criteria were patients with incomplete clinical data, the presence of synchronous/metachronous cancers, and those who underwent other major surgical interventions during the ileostomy reversal surgery.

\section{Surgical Procedure and Group Classification}

All the patients received ileostomy closure via ileostomy site incision with no laparotomy. Intravenous or oral analgesics were used for pain management after surgery without epidural analgesia. The general protocol for IV fluid administration was $1.5 \mathrm{~mL} / \mathrm{kg} / \mathrm{hr}$; however, the amount of IV fluid was also determined according to the patient's oral intake and fluid loss. Postoperative flatus is a sign of GIFR, which was prospectively recorded in realtime in the hospital information system (HIS) by nurses at our department. In this study, delayed GIFR was defined as postoperative first flatus time $\geq 3$ days after ileostomy reversal surgery.

\section{Statistical Analysis}

Data analysis was performed using the SPSS software (ver. 22.0; Chicago, IL, USA). Continuous data are expressed as the mean \pm standard deviation or median (IQR). Continuous variables and categorical variables were assessed using the $t$-tests or Mann-Whitney U. All considerable postoperative flatus-related factors were analyzed using univariate analysis; then, variables with $P<0.1$ were selected for the multivariate analysis using logistic regression. Receiver operating characteristic (ROC) curve analyses were conducted to predict the risk factors of delayed GIFR after ileostomy reversal surgery. A twosided $P<0.05$ was considered statistically significant.

\section{Results}

From January 2018 to December 2019, a total of 342 patients were screened, and 282 patients were found eligible for this study (Figure 1). Postoperative first flatus time of all patients was 3 (range, 1-9) days, and the 75th percentile of all the postoperative first flatus time was 3 days. Based on the time to first flatus classification criteria, 119 and 163 patients were classified in the early and delayed flatus group, respectively. The rate of delayed flatus was $58.8 \%$. The median length of postoperative hospital stay was 7 (range 3-23) days in the entire cohort. Compared with the early flatus group, the length of postoperative hospital stay was significantly longer in the delayed flatus group ( $7.1 \pm 2.8$ days vs $8.3 \pm 2.8$ days, 


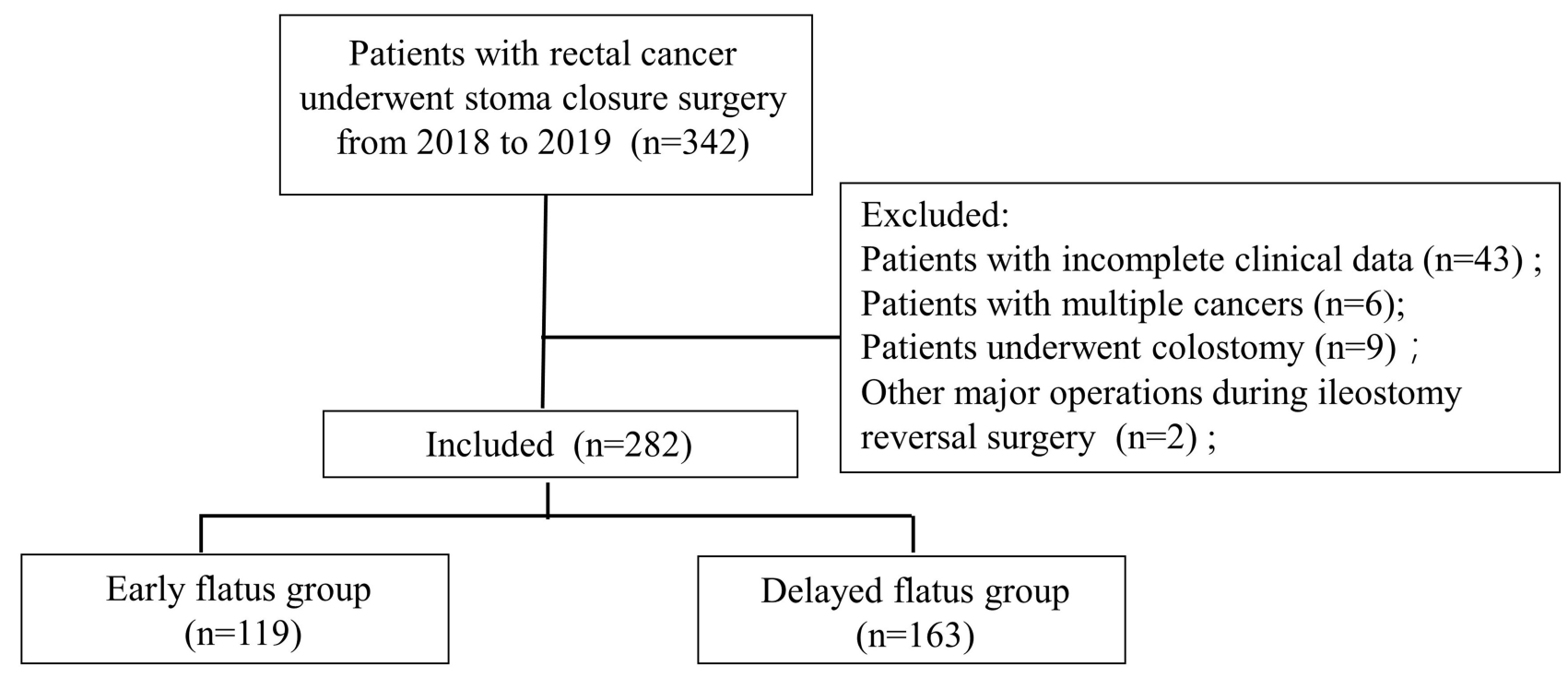

Figure I Flow chart of rectal cancer patient selection for this study.

$P<0.001)$. Three $(2.5 \%)$ patients in the early flatus group and $6(3.7 \%)$ in the delayed flatus group had postoperative complications. Postoperative complications were significantly associated with delayed postoperative flatus function $(P=0.037)$.

Table 1 illustrates the comparisons of demographic and clinical data of patients in early and delayed flatus groups. Patients in the delayed flatus group had a longer operative duration of ileostomy reversal $(P=0.022)$, more intravenous fluid infusion on postoperative day 1 (POD1) $(P<0.001)$, and longer duration of stoma ( $\geq 6$ months) $(P=0.030)$ than that of early flatus groups. Multivariate analysis (Table 2) showed that intravenous fluid infusion in POD1 (OR=1.001, 95\% CI: 1.001-1.002, $P=0.001)$ and duration of stoma $\geq 6$ months $(\mathrm{OR}=2.005,95 \% \mathrm{CI}: 1.155$ 3.657, $P=0.014$ ) were independent risk factors for delayed flatus.

ROC curve analysis was used to evaluate the potency of the prediction model in predicting the delayed flatus after ileostomy reversal surgery. As shown in Figure 2, the predictive power of the prediction model was significant, with an AUC of 0.704 (95\% CI: 0.647-0.757, $P<0.001)$. When the Hosmer-Lemeshow method was used, the value of $\mathrm{X}^{2}$ was $10.399(P>0.05)$, suggesting that the prediction model fitted well.

Figure 3 illustrates the association of delayed flatus with duration of stoma and intravenous fluid infusion. The proportions of delayed flatus in patients with duration of stoma for $<3$ months, 3 to $<6$ months, 6 to $<9$ months, and $\geq 9$ months were $54.1 \%, 52.3 \%, 64.3 \%$ and $73.1 \%$, respectively. Similarly, the proportions of delayed flatus in patients receiving fluid infusion $<1000 \mathrm{~mL}, 1000$ to $<2000 \mathrm{~mL}, 2000$ to $<3000 \mathrm{~mL}$ and $\geq 3000 \mathrm{~mL}$ were $33.3 \%, 34.0 \%, 56.1 \%$ and $82.0 \%$, respectively. The proportions of delayed flatus almost increased as the duration of stoma or intravenous fluid infusion in POD1 increased.

\section{Discussion}

In this study, we found that postoperative first flatus time ranged from 1 to 9 days and delayed flatus occurred in more than half of the patients $(58.8 \%)$ who had stoma closure surgery, which significantly increased their length of postoperative hospital stay. The median length of postoperative hospital stay after ileostomy reversal was 7 (range, 3 to 23) days and was similar to previous literature. $^{12,18}$

Ileostomy closure not only increases the patient's treatment cost, physical and mental burden but is also associated with high morbidity. In this study, the rate of postoperative complications was only $0.3 \%$. During the procedure of ileostomy reversal, most patients received gunsight sutures and stapler anastomosis, which could have decreased surgical-site infection and intestinal obstruction, thereby leading to a reduction in postoperative complications in this study. ${ }^{19,20}$ Delayed flatus may lead to increased morbidities, such as intestinal paralysis, intestinal obstruction, and more. In this present study, delayed GFIR was significantly associated with postoperative complications $(P=0.037)$. Compared with delayed GFIR group, patients in the early flatus group tended to have a reduced 
Table I Comparison of the Demographic and Clinical Characteristics

\begin{tabular}{|c|c|c|c|}
\hline Variables & $\begin{array}{c}\text { Early } \\
\text { Flatus } \\
\text { Group } \\
(n=119)\end{array}$ & $\begin{array}{c}\text { Delayed } \\
\text { Flatus } \\
\text { Group } \\
(n=163)\end{array}$ & $\mathbf{P}$ \\
\hline Age, median (IQR) & $59(49,66)$ & $6 I(53,68)$ & 0.053 \\
\hline Male, n (\%) & $82(68.9)$ & $106(65.0)$ & 0.495 \\
\hline Drinking history, n (\%) & $2(1.7)$ & I $(0.6)$ & 0.783 \\
\hline Smoking status, n (\%) & $4(3.4)$ & $7(4.3)$ & 0.930 \\
\hline Concomitant disease, n (\%) & $29(24.4)$ & $35(21.5)$ & 0.175 \\
\hline $\begin{array}{l}\text { Tumor recurrence or } \\
\text { metastasis, } n(\%)\end{array}$ & $6(5.0)$ & $7(4.3)$ & 0.767 \\
\hline $\mathrm{BMI}<18.5 \mathrm{~kg} / \mathrm{m}^{2}, \mathrm{n}(\%)$ & 14 (1I.8) & $13(8.0)$ & 0.566 \\
\hline $\begin{array}{l}\text { PTNM stages of the } \\
\text { primary tumor, } n(\%)\end{array}$ & & & 0.946 \\
\hline 0 & $12(10.1)$ & $17(10.4)$ & \\
\hline I & $33(27.7)$ & $48(29.4)$ & \\
\hline II & $43(36.1)$ & $50(30.7)$ & \\
\hline III & $25(21.0)$ & $40(24.5)$ & \\
\hline IV & $6(5.0)$ & $8(4.9)$ & \\
\hline $\begin{array}{l}\text { Distance from the primary } \\
\text { tumor to the anus }<5 \mathrm{~cm} \text {, } \\
\mathrm{n}(\%)\end{array}$ & $65(54.6)$ & $72(44.2)$ & 0.083 \\
\hline Chemotherapy, n (\%) & $75(63.0)$ & $100(61.3)$ & 0.775 \\
\hline Radiotherapy, n (\%) & $16(13.4)$ & $25(15.3)$ & 0.656 \\
\hline $\begin{array}{l}\text { Surgical approach of primary } \\
\text { tumor resection, } n(\%)\end{array}$ & & & 0.102 \\
\hline Dixon & $61(51.3)$ & $93(57.1)$ & \\
\hline Parks & $21(17.6)$ & $40(24.5)$ & \\
\hline Bacon & $4(3.4)$ & $4(2.5)$ & \\
\hline TaTME & $33(27.7)$ & $26(20.0)$ & \\
\hline $\begin{array}{l}\text { Duration of stoma } \geq 6 \\
\text { months, } \mathrm{n} \text { (\%) }\end{array}$ & $32(26.9)$ & $64(39.3)$ & 0.030 \\
\hline $\begin{array}{l}\text { Operative duration of } \\
\text { ileostomy reversal, min, } \\
\text { median (IQR) }\end{array}$ & $82(60,105.5)$ & $95(60,123)$ & 0.022 \\
\hline $\begin{array}{l}\text { Methods of anastomosis } \\
\text { Suture } \\
\text { Stapled }\end{array}$ & $\begin{array}{l}24(20.2) \\
95(79.8)\end{array}$ & $\begin{array}{l}4 \mid(25.2) \\
122(74.8)\end{array}$ & 0.326 \\
\hline $\begin{array}{l}\text { Intravenous fluid volume } \\
\text { during ileostomy reversal } \\
\text { surgery, mL, median (IQR) }\end{array}$ & $\begin{array}{c}1300(1100 \\
1600)\end{array}$ & $\begin{array}{c}1300(1100 \\
1600)\end{array}$ & 0.179 \\
\hline
\end{tabular}

(Continued)
Table I (Continued).

\begin{tabular}{|l|c|c|c|}
\hline Variables & $\begin{array}{c}\text { Early } \\
\text { Flatus } \\
\text { Group } \\
(\mathbf{n}=1 \text { 19) }\end{array}$ & $\begin{array}{c}\text { Delayed } \\
\text { Flatus } \\
\text { Group } \\
(\mathbf{n}=163)\end{array}$ & $\boldsymbol{P}$ \\
\hline $\begin{array}{l}\text { Intravenous fluid infusion in } \\
\text { PODI, mL, median (IQR) }\end{array}$ & $\begin{array}{c}2400(1900, \\
2700)\end{array}$ & $\begin{array}{c}2740(2350, \\
3070)\end{array}$ & 0.000 \\
\hline $\begin{array}{l}\text { Complications after } \\
\text { primary procedure, n (\%) }\end{array}$ & $63(52.9)$ & $72(44.2)$ & 0.145 \\
\hline
\end{tabular}

Abbreviations: PODI, postoperative day I; IQR, interquartile range.

Table 2 Multivariate Logistic Regression Analysis

\begin{tabular}{|l|c|c|c|c|}
\hline Variables & B & OR & $\begin{array}{c}\text { 95\%Cl Lower } \\
\text { Limit-Higher } \\
\text { Limit }\end{array}$ & $P$ \\
\hline $\begin{array}{l}\text { Intravenous fluid } \\
\text { infusion in PODI }\end{array}$ & 0.001 & 1.001 & $1.001-1.002$ & 0.001 \\
\hline $\begin{array}{l}\text { Duration of stoma } \\
\geq 6 \text { months }\end{array}$ & 0.720 & 2.005 & $1.155-3.657$ & 0.014 \\
\hline
\end{tabular}

Abbreviations: PODI, postoperative day I; OR, odds ratio; $\mathrm{Cl}$, confidence interval.

risk of postoperative complications, as compared to the delayed flatus group $(3.7 \%$ vs $2.5 \%, P=0.838)$.

Although intestinal continuity was restored after the ileostomy reversal surgery, postoperative GIFR of some patients was slow, which is a problem that cannot be ignored. Risk factors for the postoperative GIFG remain unclear. Interestingly, in this study, we found that intravenous fluid infusion on POD1 was an independent risk factor for the delayed flatus in the patients after ileostomy reversal surgery. All the patients received fluid infusion in POD1 according to the balance of oral intake and fluid loss. Some patients had received larger amount of fluid due to their low urine output or hypotension and the amount of fluid infusion in POD1 was variable in this study. The increased fluid infusion in POD1 resulted in prolonged flatus. Fluid management plays a very important role in improving patients' outcomes during the perioperative period. Patients receiving large volume of fluid infusion could experience fluid overload and be at increased risk of cardiac preload and postoperative complications, such as AL, pulmonary edema, pneumonia, and wound infection. ${ }^{15,21}$ Nisanevich et al showed that restrictive fluid infusion could lead to earlier time to flatus and 


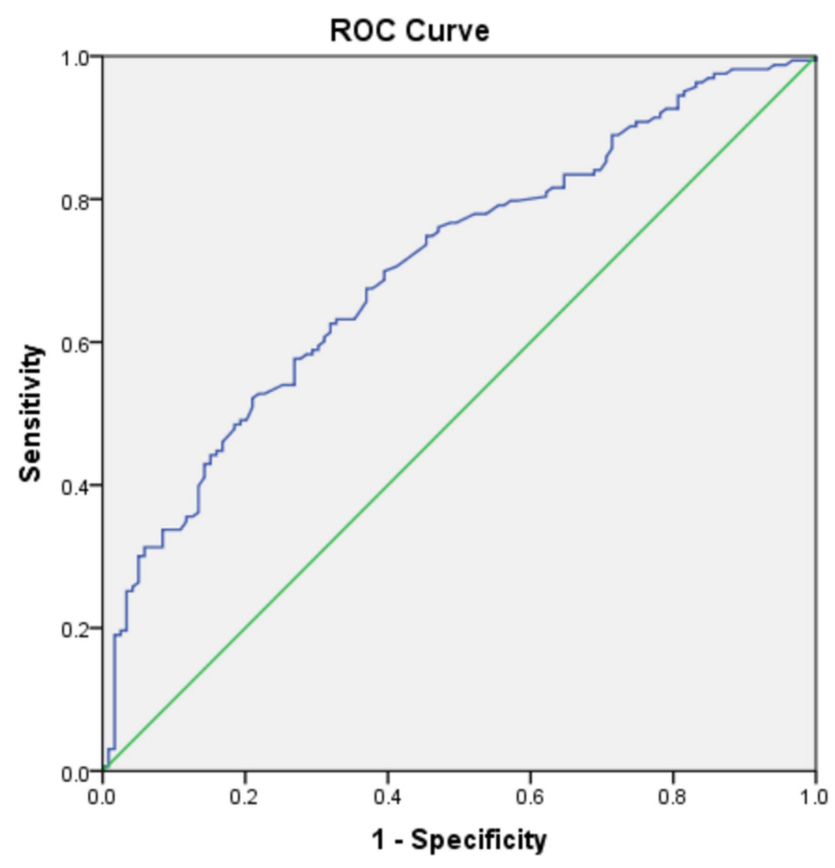

Figure 2 ROC curve of a new model for predicting the delayed flatus after ileostomy reversal in rectal cancer patients ROC AUC was $0.704(95 \% \mathrm{Cl}$ : $0.647-$ $0.757, P<0.00 \mathrm{I})$. When the Hosmer-Lemeshow method was used, the value of $X^{2}$ was $10.399, P>0.05$.

Abbreviations: ROC, receiver operating characteristic; AUC, area under the receiver-operating characteristic curve.

bowel movement, fewer immediate complications, and length of hospital stay. ${ }^{22}$ Early oral intake in the postoperative period to reduce the intravenous fluid infusion should be encouraged. Early enteral nutrition as reported can facilitate the improvement of nutritional status, further enhancement of GIFR, reduce the risk of complications and shorten postoperative hospital stay. ${ }^{23-26}$

The duration from the primary operation to the restoration of gastrointestinal tract continuity may vary considerably as it depends on the tumor status, pelvic floor function and the patient's condition. ${ }^{27}$ The present study showed that the average time between the primary operation and the closure of the ileostomy was 171 days, which was similar to a study by Law et al (183 days). ${ }^{28}$ Furthermore, in this study, we found that the duration of stoma $\geq 6$ months was another independent risk factor for delayed flatus. Previously, it was reported that diverting stoma was associated with bowel dysfunction, which may be due to alterations in colonic nutrition leading to inflammation, changes in the bacterial flora, and/or atrophy of motility or sensory elements. ${ }^{29-31}$ The rate of diversion colitis is reported to be $70 \%$ to $100 \%$ in Western countries who underwent diversion ostomy. ${ }^{32}$ Recent studies reported that the long duration of stoma $\geq 6$ months was associated with a 3.7-fold increase in the risk of major bowel dysfunction after the restoration of bowel continuity. $^{33,34}$ Therefore, this could explain the association of longer duration of stoma to cause prolonged GIFR found in this study. And it was reported that early closure is a safe and effective therapeutic approach, improving the recovery. ${ }^{35}$ In addition, the operative duration of ileostomy reversal in the delayed flatus group was significantly longer than in the early flatus group, which was consistent with a previous report which showed that the first flatus was detected earlier in the shorter operative duration group. $^{36}$ However, the operative duration of ileostomy reversal was not an independent risk factor for delayed flatus in this study.

The main limitations of this study were its retrospective design, single-center data and sample size, which could have led to a certain level of inevitable bias. A prospective, multi-center study with larger sample size
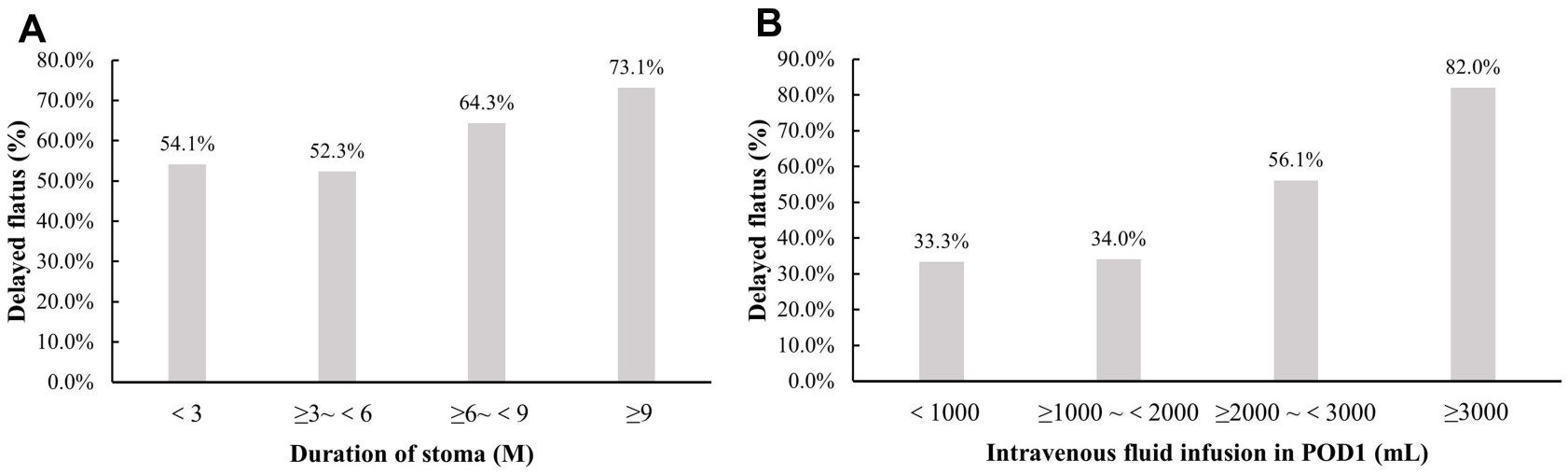

Figure 3 Association of delayed flatus with duration of stoma (A) or fluid infusion (B) the proportions of delayed flatus almost increased as the duration of stoma or intravenous fluid infusion PODI increased.

Abbreviation: PODI, postoperative day I. 
should be conducted to confirm our findings to better guide the treatment and improve the recovery of these patients.

\section{Conclusion}

In this study, we observed that the incidence of delayed flatus was $58.8 \%$, and increased intravenous fluid infusion at POD1 and duration of stoma $\geq 6$ months were independently associated with delayed flatus for patients undergoing ileostomy reversal surgery. Larger size cohort and prospective settings are needed to confirm whether a reduction in intravenous fluid infusion on POD1 and shorter duration of stoma could improve treatment outcomes of rectal cancer patients.

\section{Abbreviations}

GIFR, gastrointestinal function recovery; ERAS, enhanced recovery after surgery; AL, Anastomotic leakage; POD1, postoperative day 1; ROC, receiver operating characteristic; AUC, area under the receiver-operating characteristic curve; IQR, interquartile range; $\mathrm{OR}$, odds ratio; $\mathrm{CI}$, confidence interval.

\section{Data Sharing Statement}

All datasets used and/or analyzed during the current study are available from the corresponding author on reasonable request.

\section{Ethics Statement}

This study was approved by the Ethical Committee of the Sixth Affiliated Hospital of Sun Yat-sen University (No. 2020ZSLYEC-139) and was conducted in accordance with the provisions of the World Medical Association's Declaration of Helsinki of 1995 (revised in Tokyo, 2004). Because this was a retrospective study, informed consent was waived.

\section{Acknowledgments}

We would like to extend our sincere gratitude to every researcher and participant who contributed to this study. This study was supported by Sun Yat-sen University Clinical Research 5010 Program (Grant Number: 2019021), National Natural Science Foundation of China (Grant Number: 81803163) and Natural Science Foundation of Guangdong Province (Grant Number: 2018A030310319). The funders had no role in the study design, data collection and analysis, decision to publish, or preparation of the manuscript. Lili Chu, Hui Wang and
Suyu Qiu are co-first authors for this study. Xiaoyan Huang and Rongkang Huang have contributed equally as corresponding authors.

\section{Author Contributions}

All authors made a significant contribution to the work reported, whether that is in the conception, study design, execution, acquisition of data, analysis and interpretation, or in all these areas; took part in drafting, revising or critically reviewing the article; gave final approval of the version to be published; have agreed on the journal to which the article has been submitted; and agree to be accountable for all aspects of the work.

\section{Disclosure}

The authors report no conflicts of interest in this work.

\section{References}

1. Sung H, Ferlay J, Siegel RL, et al. Global cancer statistics 2020: GLOBOCAN estimates of incidence and mortality worldwide for 36 cancers in 185 countries. CA Cancer J Clin. 2021;71(3):209-249. doi: $10.3322 /$ caac. 21660

2. Siegel RL, Miller KD, Jemal A. Cancer statistics, 2019. CA Cancer J Clin. 2019;69(1):7-34. doi:10.3322/caac.21551

3. Keller DS, Berho M, Perez RO, et al. The multidisciplinary management of rectal cancer. Nat Rev Gastroenterol Hepatol. 2020;17 (7):414-429. doi:10.1038/s41575-020-0275-y

4. Koedam TWA, Bootsma BT, Deijen CL, et al. Oncological outcomes after anastomotic leakage after surgery for colon or rectal cancer: increased risk of local recurrence. Ann Surg. 2020. doi:10.1097/ SLA.0000000000003889

5. Huang J, Liu J, Fang J, et al. Identification of the surgical indication line for the Denonvilliers' fascia and its anatomy in patients with rectal cancer. Cancer Commun (Lond). 2020;40(1):25-31. doi:10. $1002 / \mathrm{cac} 2.12003$

6. Rombey T, Panagiotopoulou IG, Hind D, Fearnhead NS. Preoperative bowel stimulation prior to ileostomy closure to restore bowel function more quickly and improve postoperative outcomes: a systematic review. Colorectal Dis. 2019;21(9):994-1003. doi:10.1111/codi.14636

7. Beirens K, Penninckx F; Procare. Defunctioning stoma and anastomotic leak rate after total mesorectal excision with coloanal anastomosis in the context of PROCARE. Acta Chir Belg. 2012;112 (1):10-14. doi:10.1080/00015458.2012.11680789

8. Chude GG, Rayate NV, Patris V, et al. Defunctioning loop ileostomy with low anterior resection for distal rectal cancer: should we make an ileostomy as a routine procedure? A prospective randomized study. Hepatogastroenterology. 2008;55(86-87):1562-1567.

9. Gessler B, Haglind E, Angenete E. Loop ileostomies in colorectal cancer patients--morbidity and risk factors for nonreversal. $J$ Surg Res. 2012;178:708-714. doi:10.1016/j.jss.2012.08.018

10. Chow A, Tilney HS, Paraskeva P, et al. The morbidity surrounding reversal of defunctioning ileostomies: a systematic review of 48 studies including 6107 cases. Int $J$ Colorectal Dis. 2009;24 (6):711-723. doi:10.1007/s00384-009-0660-z

11. Delaney CP, Senagore AJ, Viscusi ER, et al. Postoperative upper and lower gastrointestinal recovery and gastrointestinal morbidity in patients undergoing bowel resection: pooled analysis of placebo data from 3 randomized controlled trials. Am J Surg. 2006;191 (3):315-319. doi:10.1016/j.amjsurg.2005.10.026 
12. Mengual-Ballester M, Garcia-Marin JA, Pellicer-Franco E, et al. Protective ileostomy: complications and mortality associated with its closure. Rev Esp Enferm Dig. 2012;104(7):350-354. doi:10.4321/S1130-01082012000700003

13. Robertson JP, Wells CI, Vather R, Bissett IP. Effect of diversion ileostomy on the occurrence and consequences of chemotherapy-induced diarrhea. Dis Colon Rectum. 2016;59 (3):194-200. doi:10.1097/DCR.0000000000000531

14. Fiore JF, Browning L, Bialocerkowski A, Gruen RL, Faragher IG, Denehy L. Hospital discharge criteria following colorectal surgery: a systematic review. Colorectal Dis. 2012;14(3):270-281. doi:10. 1111/j.1463-1318.2010.02477.x

15. Bazargani ST, Ghodoussipour S, Tse B, et al. The association between intraoperative fluid intake and postoperative complications in patients undergoing radical cystectomy with an enhanced recovery protocol. World J Urol. 2018;36(3):401-407. doi:10.1007/s00345017-2164-8

16. Tahir W, Bolton W, Pericleous A, et al; Yorkshire Surgical Research Collaborative. Multicentre observational study of gastrointestinal recovery after elective colorectal surgery. Colorectal Dis. 2018;20 (6):536-544. doi:10.1111/codi.13949

17. Ljungqvist $\mathrm{O}$, Scott M, Fearon KC. Enhanced recovery after surgery: a review. JAMA Surg. 2017;152(3):292-298. doi:10.1001/jamasurg. 2016.4952

18. Sier MF, van Gelder L, Ubbink DT, et al. Factors affecting timing of closure and non-reversal of temporary ileostomies. Int $J$ Colorectal Dis. 2015;30(9):1185-1192. doi:10.1007/s00384-015-2253-3

19. Hsieh MC, Kuo LT, Chi CC, et al. Pursestring closure versus conventional primary closure following stoma reversal to reduce surgical site infection rate: a meta-analysis of randomized controlled trials. Dis Colon Rectum. 2015;58(8):808-815. doi:10.1097/DCR.00 00000000000401

20. Li C-K, Liang -W-W, Wang H-M, et al. Gunsight sutures significantly reduce surgical-site infection after ileostomy reversal compared with linear sutures. Gastroenterol Rep. 2020. doi:10.1093/gastro/goaa075

21. Brandstrup B, Tonnesen H, Beier-Holgersen R, et al. Effects of intravenous fluid restriction on postoperative complications: comparison of two perioperative fluid regimens: a randomized assessor-blinded multicenter trial. Ann Surg. 2003;238(5):641-648. doi:10.1097/01.sla.0000094387.50865.23

22. Nisanevich V, Felsenstein I, Almogy G, et al. Effect of intraoperative fluid management on outcome after intraabdominal surgery. Anesthesiology. 2005;103(1):25-32. doi:10.1097/00000542-2005 07000-00008

23. Pędziwiatr M, Mavrikis J, Witowski J, et al. Current status of enhanced recovery after surgery (ERAS) protocol in gastrointestinal surgery. Med Oncol. 2018;35(6):95. doi:10.1007/s12032-018-1153-0

24. Nanthakumaran S, Heys SD. Randomized clinical trial of the effects of preoperative and postoperative oral nutritional supplements on clinical course and cost of care. Br J Surg. 2004;91(11):1528. doi:10.1002/bjs.4853
25. Qiang H, Yuanshui S. Effect of early enteral nutrition on laparoscopic common bile duct exploration with enhanced recovery after surgery protocols. Eur J Clin Nutr. 2020;74(4):666. doi:10.1038/s41430-0200565-Z

26. Herbert G, Perry R, Andersen HK, et al. Early enteral nutrition within 24 hours of lower gastrointestinal surgery versus later commencement for length of hospital stay and postoperative complications. Cochrane Database Syst Rev. 2019;7:CD004080. doi:10.1002/ 14651858.CD004080.pub4

27. Zielinski T, Czyzewski P, Szczepkowski M. The usefulness of anorectal manometry in patients with a stoma before and after surgery to restore the continuity of the gastrointestinal tract. Pol Przegl Chir. 2016;88(1):1-6. doi:10.1515/pjs-2016-0019

28. Law WL, Chu KW, Choi HK. Randomized clinical trial comparing loop ileostomy and loop transverse colostomy for faecal diversion following total mesorectal excision. Br J Surg. 2002;89(6):704-708. doi:10.1046/j.1365-2168.2002.02082.x

29. Keane C, Park J, Oberg S, et al. Functional outcomes from a randomized trial of early closure of temporary ileostomy after rectal excision for cancer. Br J Surg. 2019;106(5):645-652. doi:10.1002/ bjs. 11092

30. Jimenez-Gomez LM, Espin-Basany E, Trenti L, et al. Factors associated with low anterior resection syndrome after surgical treatment of rectal cancer. Colorectal Dis. 2017. doi:10.1111/codi.13901

31. Diao F, Cai S. Aspirin-based chemoprevention of colorectal cancer: the role for gut microbiota. Cancer Commun (Lond). 2020;40 (11):633-635. doi:10.1002/cac2.12086

32. Ferguson CM, Siegel RJ. A prospective evaluation of diversion colitis. Am Surg. 1991;57(1):46-49.

33. Hughes DL, Cornish J, Morris C; Group LTM. Functional outcome following rectal surgery-predisposing factors for low anterior resection syndrome. Int $J$ Colorectal Dis. 2017;32(5):691-697. doi:10.1007/s00384-017-2765-0

34. Vaughan-Shaw PG, Gash K, Adams K, et al. Protocol for a multicentre, dual prospective and retrospective cohort study investigating timing of ileostomy closure after anterior resection for rectal cancer: the CLOSurE of Ileostomy Timing (CLOSE-IT) Study. BMJ Open. 2018;8(10):e023305. doi:10.1136/bmjopen-2018-023305

35. Kłęk S, Pisarska M, Milian-Ciesielska K, et al. Early closure of the protective ileostomy after rectal resection should become part of the Enhanced Recovery After Surgery (ERAS) protocol: a randomized, prospective, two-center clinical trial. Wideochir Inne Tech Malo Inwazyjne. 2018;13(4):435-441. doi:10.5114/wiitm.2018.79574

36. Sisik A, Erdem H. Effect of trocar site bupivacaine administration, time of first passage of flatus, and duration of the surgery on postoperative pain after sleeve gastrectomy: a Case Control Study. Obes Surg. 2019;29(2):444-450. doi:10.1007/s11695-018-3529-9
Cancer Management and Research is an international, peer-reviewed open access journal focusing on cancer research and the optimal use of preventative and integrated treatment interventions to achieve improved outcomes, enhanced survival and quality of life for the cancer patient.
The manuscript management system is completely online and includes a very quick and fair peer-review system, which is all easy to use. Visit http://www.dovepress.com/testimonials.php to read real quotes from published authors. 